\title{
Fluorescence detection system based on silicon quantum dots-polysilane nanocomposites
}

\author{
L. Sacarescu*, G. Roman, G. Sacarescu, M. Simionescu \\ Petru Poni Institute of Macromolecular Chemistry, Aleea Grigore Ghica Voda 41A, 700487 Iasi, Romania
}

Received 4 May 2016; accepted in revised form 16 July 2016

\begin{abstract}
A dual channel fluorescence system that combines the optical properties of silicon quantum dots-polysilane nanocomposites with those of 2-(4-chlorophenyl)-6-(thiophen-2-yl)pyridine, a fluorescent cytotoxic agent, is presented. The system is capable to alternatively trigger emission signals at two different wavelengths by excitation at a single wavelength. For this purpose a highly stable colloidal dispersion of silicon quantum dots-polysilane nanocomposite is prepared by a one-pot synthetic method using microwave-activated Wurtz coupling of organochlorosilanes. The size and shape of the silicon quantum dots within the polysilane thin film are studied by TEM. The colloidal dispersions are investigated by SAXS, which evidences that polysilane plays also a role as stabilizing agent to prevent aggregation. UV-vis spectrophotometry of the silicon quantum dots-polysilane nanocomposites in the presence of 2-(4-chlorophenyl)-6-(thiophen-2-yl)pyridine is used to define the active wavelength range and establish the fluorescence detection method.
\end{abstract}

Keywords: polymer composites, nanomaterials, quantum dots, chemical synthesis, optical properties

\section{Introduction}

During the last decade, intense efforts have been undertaken to miniaturize optical and electronic devices while increasing their efficiency [1-3]. Nowadays, the development of nano and quantum devices for high tech applications is close to go beyond the fundamental conceptual stage. From this point of view an attractive and still unexplored domain is related to systems were inorganic nanoparticles interact with organic molecules or polymers having specific optoelectronic properties [4-6]. The variety of particles and polymers having exciting optical and/or electronic properties continuously grows, and several of them seem to have the necessary potential toward new developments [7-10]. This work aims at showing the interesting optical properties that could be obtained when both the inorganic nanoparticles and polymer are silicon-based structures.
Quantum dots are promising materials for a wide range of applications related to quantum electronics [11-14]. Silicon itself represents a special case amongst the semiconductive materials used to obtain quantum dots. An important advantage of silicon quantum dots ( $\mathrm{SiQDs}$ ) is that, unlike other elements, silicon is biocompatible. Consequently, SiQDs can be used in hot research fields of nanomedicine, such as fluorescent sensing, labelling and dynamic therapy [15-18]. On the other hand, SiQDs still undergo intensive research, since their fundamental photophysics is less understood than the photophysics of particles made from direct gap semiconductors [19]. In addition, obtaining SiQDs in a controlled manner is still a challenge.

Within the class of silicon-based conjugated polymers, polysilanes present interesting optoelectronic properties owing to their $\sigma$ conjugated chains [20, 21]. An increase in the substitution of the silicon

\footnotetext{
*Corresponding author, e-mail: livius@icmpp.ro C BME-PT
} 
chain in polysilanes with aromatic moieties leads to further enhancement of their optoelectronical properties, owing to the intermingling of $\sigma$ and $\sigma-\pi$ conjugation. This extended conjugation also makes polysilanes sensitive towards light, but this disadvantage could be turned into an advantage in applications such as micro- and nanolithography [22-24]. In addition, polysilanes have interesting non-linear optical (NLO) properties, and they possess intrinsic semiconductivity [25-31].

Polyhydrosilanes are a distinct class of polyorganosilane polymers containing hydrosilyl moieties [32]. The properties of these functional silicon-based macromolecular structures are different from those of poly(diorganosilylene)s [33, 34]. Due to the reactive methylhydrosilyl segments, such materials have been used to generate in situ important amounts of silicon nanoparticles [35]. Steady-state fluorescence analysis showed that the presence of the silicon nanoparticles within the polysilane matrix leads to characteristic modifications in the emission spectrum profile [36].

The current study describes a one-pot synthetic method and the optical properties of silicon quantum dots-polysilane nanocomposites (NQD). Subsequently, the dual channel fluorescence of NQD in the presence of the antitumoral agent 2-(4-chlorophenyl)-6(thiophen-2-yl)pyridine (CTP) is studied. Such materials have a high potential for applications in the field of nanomedicine as molecular contrasting agents in imaging and therapy of tumours.

\section{Experimental}

\subsection{Materials}

Dichlorodiphenylsilane, $\left(\mathrm{C}_{6} \mathrm{H}_{5}\right)_{2} \mathrm{SiCl}_{2},(>98 \%, 42930)$ and dichloromethylsilane (>98\%, 440248), $\left(\mathrm{CH}_{3}\right) \mathrm{HSiCl}_{2}$, were purchased from Sigma-Aldrich Chemie $\mathrm{GmbH}$, Germany and distilled prior to use. Toluene (244511) and tetrahydrofuran (THF, 401757) were purchased from Aldrich and used after distillation over sodium wire under nitrogen atmosphere. 2-Acetylthiophene (W503509), paraformaldehyde (P6148), dimethylamine hydrochloride (38960), 2bromo-1-(4-chlorophenyl)ethanone (101273), ammonium acetate (A7330), pyridine (270970) and the solvents required for the synthesis of CTP were obtained from Sigma-Aldrich Chemie GmbH, Germany and were used as such. 3-Dimetylamino-1-(thiophen- 2-yl)propan-1-one hydrochloride and 1-(2-(4-chlorophenyl)-2-oxoethyl)pyridinium bromide were prepared as already described in a previously published article [37].

Poly[diphenyl-co-methyl $(\mathrm{H})]$ silane copolymer, molar ratio $\left(\mathrm{C}_{6} \mathrm{H}_{5}\right)_{2} \mathrm{Si} / \mathrm{CH}_{3}(\mathrm{H}) \mathrm{Si}$ 1:1, was obtained in a closed reaction system using a Discover Labmate microwave reactor (CEM Corporation, USA) equipped with an infrared temperature probe and a magnetic stirrer [38]. The reactor was operated in standard conditions under the control of its Synergy software, by employing temperature as main parameter.

\subsection{Synthesis of NQD}

A simple, one-pot method to obtain soluble polysilane-silicon nanoparticles composite has been described previously [39]. This approach is based on the Wurtz coupling of methyldichlorosilane with various organodichlorosilanes in heterogeneous conditions. Thus, beside the polyhydrosilane copolymer, silicon nanoparticles are present in the final reaction product. In order to increase the content in silicon nanoparticles within the polymer matrix, a modified synthetic procedure is presented in this work. This time the coupling reaction is performed under microwaves irradiation.

A $10 \mathrm{~mL}$ microwave vial containing a magnetic stirring bar was flushed with dry nitrogen at $80^{\circ} \mathrm{C}$ for $30 \mathrm{~min}$, then a mixture of diphenyldichlorosilane $(1.5 \mathrm{~g}, 6 \mathrm{mmol})$ and methyl $(\mathrm{H})$ dichlorosilane $(0.7 \mathrm{~g}$, $6 \mathrm{mmol})$ in $2 \mathrm{~mL}$ dry toluene was added. Next, a dispersion of sodium $(0.5 \mathrm{~g})$ in toluene $(2 \mathrm{~mL})$ was carefully added, and then the reaction mixture was degassed by ultrasonication under dry nitrogen. The microwave reactor setup was in standard mode, close vessel conditions, with the following parameters: power $=50 \mathrm{~W}$; temperature $=120^{\circ} \mathrm{C}$; time $=10 \mathrm{~min}$. The exothermal coupling reaction started almost instantly, and the temperature rose quickly to approx. $100^{\circ} \mathrm{C}$. At the end of the reaction time, the dark purple mixture was cooled to room temperature before it was transferred into a $50 \mathrm{~mL}$ flask. Dry toluene $(10 \mathrm{~mL})$ was added, and the unreacted sodium was quenched carefully, under efficient stirring, using $10 \mathrm{~mL}$ of ethanol. The organic layer was washed with distilled water until neutral $\mathrm{pH}$ and subsequently dried over anhydrous $\mathrm{MgSO}_{4}$. Toluene was removed 
under reduced pressure, and then THF $(1 \mathrm{~mL})$ was added to dissolve the solid material. The solution was poured onto $100 \mathrm{~mL}$ methanol, and the resulting white precipitate was filtered and vacuum dried at $50{ }^{\circ} \mathrm{C}$ overnight to afford NQD as a powder $(1.7 \mathrm{~g}, 75 \%)$. ${ }^{1} \mathrm{H}-\mathrm{NMR}\left(400 \mathrm{MHz}, \mathrm{CDCl}_{3}, \delta\right): 0.48\left(\mathrm{Si}-\mathrm{CH}_{3}\right), 4.7$ ( $\mathrm{Si}-\mathrm{H}), 7.28\left(\mathrm{Si}-\mathrm{C}_{6} \mathrm{H}_{5}\right)$. FTIR $\left(\mathrm{KBr}, \mathrm{cm}^{-1}\right): 3064$ and $3045\left(\mathrm{C}-\mathrm{H}_{\text {arom }}\right), 2953$ and $2893\left(\mathrm{C}-\mathrm{H}_{\text {aliph }}\right), 2100$ $(\mathrm{Si}-\mathrm{H}), 1424$ and $1096\left(\mathrm{Si}-\mathrm{C}_{6} \mathrm{H}_{5}\right), 1243\left(\mathrm{Si}-\mathrm{CH}_{3}\right)$, 695 (Si-C), 461 (Si-Si). GPC (SLS-MALS, 1 mg/mL in THF): $M_{\mathrm{w}}=4495 \mathrm{~mol} \cdot \mathrm{g}^{-1} ; M_{\mathrm{w}} / M_{\mathrm{n}}=1.5$.

\subsection{Synthesis of 2-(4-chlorophenyl)-6- (thiophen-2-yl)pyridine (CTP)}

CTP was synthesized using a previously reported procedure through the condensation of 3-dimetylamino-1-(thiophen-2-yl)propan-1-one hydrochloride 1, 1-(4-chlorophenacyl) pyridinium bromide 2 and ammonium acetate, and obtained as colorless crystals, mp $88-89^{\circ} \mathrm{C} .{ }^{1} \mathrm{H}-\mathrm{NMR}\left(400 \mathrm{MHz}, \mathrm{CDCl}_{3}\right.$,

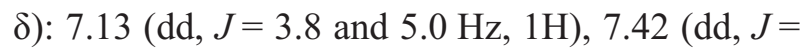
1.0 and $5.0 \mathrm{~Hz}, 1 \mathrm{H}), 7.46(\mathrm{~d}, J=8.8 \mathrm{~Hz}, 2 \mathrm{H}), 7.54$ $7.61(\mathrm{~m}, 2 \mathrm{H}), 7.65(\mathrm{dd}, J=1.0$ and $3.8 \mathrm{~Hz}, 1 \mathrm{H}), 7.74$ $(\mathrm{t}, J=8.0 \mathrm{~Hz}, 1 \mathrm{H}), 8.06(\mathrm{~d}, J=8.8 \mathrm{~Hz}, 2 \mathrm{H})$. ${ }^{13} \mathrm{C}$-NMR (400 MHz, $\mathrm{CDCl}_{3}, \delta$ ): 117.3, 118.1, 124.8, 127.9, 128.1, 128.3, 129.0, 135.3, 137.5, 137.7, 145.4, 152.5, 155.6. Anal. calcd. for $\mathrm{C}_{15} \mathrm{H}_{10} \mathrm{ClNS}$ : C 66.29, H 3.71, N 5.15; found: C 66.41, H 3.62, N 5.27 [37].

\section{Measurements}

${ }^{1} \mathrm{H}$ - and ${ }^{13} \mathrm{C}$-NMR spectra were recorded at room temperature using a Bruker DRX $400 \mathrm{MHz}$ NMR instrument (Bruker Corporation, Germany). In the case of CTP, the signals owing to the residual protons in $\mathrm{CDCl}_{3}$ were used as internal standard for the ${ }^{1} \mathrm{H}-\mathrm{NMR}$ spectrum, while the chemical shifts for the carbon atoms in the ${ }^{13} \mathrm{C}$-NMR spectrum are given relative to residual $\mathrm{CHCl}_{3}(\delta=77.16 \mathrm{ppm})$. Gel permeation chromatography (GPC) analysis was performed in THF solution at $25^{\circ} \mathrm{C}$ and at a flow rate of $1 \mathrm{~mL} / \mathrm{min}$ using a WGE SEC/GPC multidetection chromatograph (WGE Dr Bures GmbH\&Co KG, Germany) equipped with two Agilent PLgel $5 \mu \mathrm{m}$ MIXED columns (103 and $500 \AA$ ). Transmission electron microscopy (TEM) analysis was performed with a Hitachi HT7700 microscope (Hitachi High-Technolo- gies Corporation, Japan) operated in high-contrast mode at $100 \mathrm{kV}$ accelerating voltage. The microscope was used to examine the samples in High Resolution/High Contrast, Diffraction and STEM-EDX modes. The samples were prepared by drop casting the solution directly on the TEM grids (Ted Pella, 300 mesh, carbon layer) and they were subsequently kept in a box saturated with solvent vapors for $24 \mathrm{~h}$. Finally, the box was slowly flushed with nitrogen for another $24 \mathrm{~h}$, and the samples were dried under vacuum at room temperature for $60 \mathrm{~h}$. TEM analysis was performed at $-190^{\circ} \mathrm{C}$ in order to avoid the thermal destruction of the samples. Small-angle X-ray scattering (SAXS) measurements were carried out using a Bruker NanostarU instrument (Bruker Corporation, Germany) equipped with a I $\mu \mathrm{S}$ microsource with copper anode and a three-pinhole collimation system. A highly sensitive 2D detector (Vantec2000), having $68 \mu \mathrm{m}$ resolution, was used to record the scattered intensity. The scattered intensity $I(q)$ is measured as a function of the momentum transfer vector $q=4 \pi \sin \theta / \lambda$, where $\lambda$ is the wavelength of the $\mathrm{X}$-rays ( $\mathrm{Cu} \mathrm{K} \alpha$ radiation, $1.54 \AA$ ), and $\theta$ is half the scattering angle. The sample-to-detector distance was $107 \mathrm{~cm}$, allowing measurements with $q$ values between 0.008 and $0.3 \AA^{-1}$. The angular scale was calibrated by the scattering peaks of a silver behenate standard. Solutions of the samples under study were sealed in a quartz capillary and measured under vacuum at constant temperature, $25^{\circ} \mathrm{C}$ for $8000 \mathrm{~s}$. The raw data was normalized for the transmission coefficient and the incoherent scattering due to the capillary and solvent backgrounds were subtracted in the data analysis using the SAXS-Bruker AXS software. Data analysis was done using ATSAS 2.5.1 package and DIFFRAC ${ }^{\text {plus }}$ NanoFit [40]. Electronic absorption spectra (UV-vis) were recorded using a UV 7100 spectrophotometer (Shimadzu, Japan), while fluorescence spectra were obtained on a Horiba FluoroMax-4 spectrofluorometer (Horiba Scientific, Japan). All spectroscopic measurements were performed in solution at room temperature after the samples were deaerated thoroughly with nitrogen for 20 min. Fluorescence spectra were recorded by exciting the investigated materials at their corresponding absorption maxima. 


\section{Results and discussion}

\subsection{Morphologies and structures}

The synthesis of NQD has been performed by the heterogeneous Wurtz coupling of equimolar amounts of diphenyldichlorosilane and methyl(H)dichlorosilane using microwaves. The reaction takes place in toluene solvent in the presence of dispersed sodium. The process is complex and the influence of microwaves is reflected especially in the effects on the reaction time, the yield and the content of silicon particles in the nanocomposite. In the current approach microwaves were used at a considerably low power. Thus, the action of microwaves was strong enough to act upon the polar species present in the reaction system, while being too weak to initiate secondary reactions. Figure 1 shows the mechanism through which the main intermediates responsible for the generation of SiQDs arise from the thermal cleavage of the methylhydrosilyl segments. These silylenes are prone to be affected by microwaves, and they accelerate the chemo-restructuration processes that finally lead to formation of SiQDs (Figure 1). This process runs in competition with the main Wurtz coupling reaction and lead to scission of some of polyhydrosilane chains into low molecular weight species. These are soluble in methanol and are eliminated during purification. Through this approach, relative large quantities of NQD could be obtained easily in a sequence of experiments under perfectly controlled conditions. While the microwave-assisted method ensures a high reproducibility of the process, it has always been difficult to obtain batches of composites with similar characteristics using conventional heating [39]. Another important advantage of the method is that it produces in situ a solid state precursor for colloidal SiQDs dispersions. Such colloidal dispersions can be obtained by simply dissolving NQD in common organic solvents (xylenes, toluene, tetrahydrofuran). Due to the specific interfacial properties, polyhydrosilane acts like a stabilizing agent for SiQDs, and these dispersions are highly stable. For example, samples kept in the dark at room temperature and under an inert atmosphere preserve their properties and appearence unaltered, and they can be used to produce thin films on various supports.

The polymeric matrix resulted from this synthesis is a polyhydrosilane copolymer with a statistical distribution of methylhydrosilyl and diphenylsilyl segments. The molar rate for these two structural units was $M_{\mathrm{r}}=1: 0.8$, as estimated by ${ }^{1} \mathrm{H}-\mathrm{NMR}$. As shown previously, the main characteristic of this material is the presence of silicon nanoparticles that are dispersed within the solid polymer $[35,36,39]$. The ex-
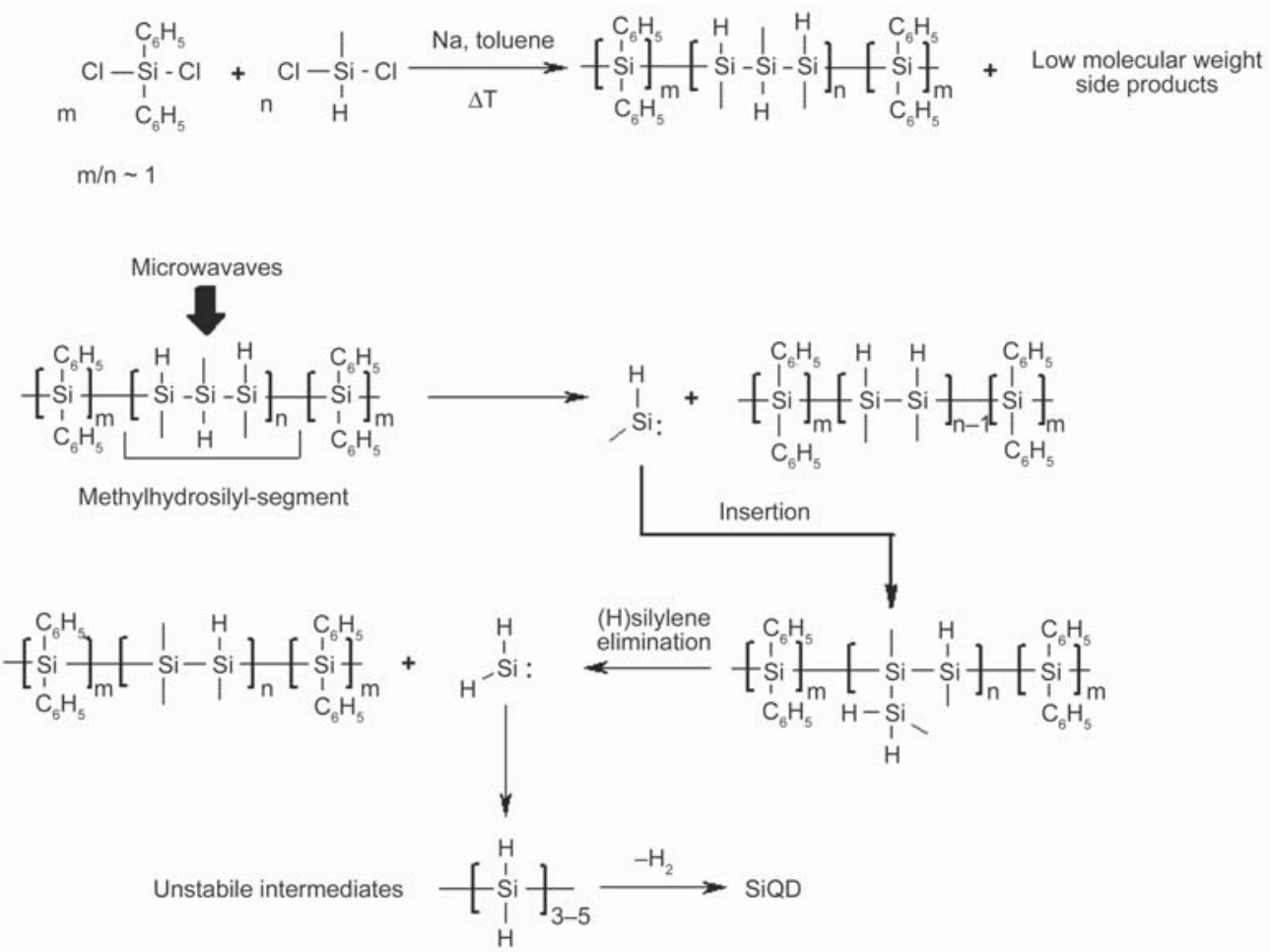

Figure 1. Synthesis of NQD showing formation of SiQDs 


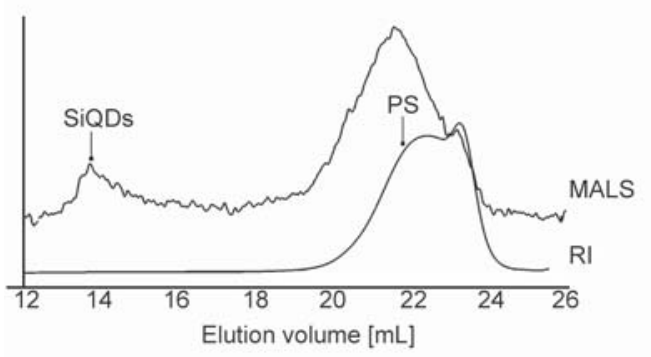

Figure 2. GPC comparison of MALS to RI traces that evidence existence of the SiQDs peak

istence of these nanoparticles can be also inferred from GPC analysis. Thus, beside the peak representing the polysilane molecular weight distribution, the MALS (multi angle light scattering) detector clearly evidenced another, unusual and low intensity peak at $14 \mathrm{~mL}$ elution volume. It is important to note that this peak is not detected by the refractive index (RI) detector (Figure 2). The difference should be related to the detection principle of the instruments. Since the low intensity peak is present only in MALS trace then the best guess is to assign it to the presence of solid particles within the liquid sample flow. Further investigations were meant to ascertain their specific characteristics as SiQDs.

In this regard, the TEM images provided clarifying details concerning the thin layer of the composite. Thus, well-defined nanoparticles dispersed within the bulk material can be noticed at high magnification (Figure 3). The nanoparticles were spherical and polydisperse, with average dimensions within the 3 $30 \mathrm{~nm}$ range (Figure $3 \mathrm{~b}$ ). STEM images showed that

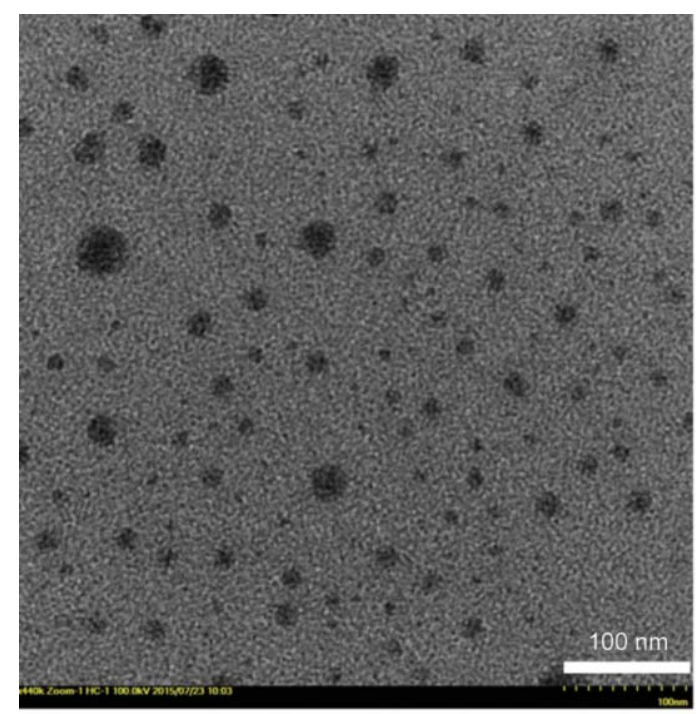

a) the particles are compact, with an even distribution of highly dense material in all directions (Figure 4). Obviously, the particles are covered by a polysilane layer. However, the EDX line profile analysis indicates that, compared with the background, silicon is the main constitutive element of the observed nanoparticles. Furthermore, in order to have insights concerning the particle structure, the selected-area electron diffraction (SAED) pattern was investigated (Figure $4 \mathrm{~b}$ ). Thus, there are three diffraction rings that confirm the crystalline nature of the nanoparticles.

The disadvantage of TEM imaging is that it shows details on a very small fraction of the sample which is deposed on the grid surface. In order to obtain more reliable information the average dimensions and shape of the SiQDs in NQD colloidal dispersion were determined by SAXS analysis. Diluted samples of NQD have been submitted to X-ray exposure.

The collected 2D patterns were further reduced by azimuthal integration to $1 \mathrm{D}$ plots where the intensity of the scattering, $I$, is printed versus the momentum transfer vector, $q$ (Figure 5). It is known that the scattering intensity $I(q)$ is related to the averaged ensemble form of the particles. The intraparticle effects can be described by the so called form factor $P(q)$, while the interparticle ones effects can be expressed by the structure factor $S(q)$.

In the case of diluted systems, $S(q)$ could be neglected. Both $P(q)$ and $S(q)$ are interrelated and described by the variation of scattering intensity with the mo-

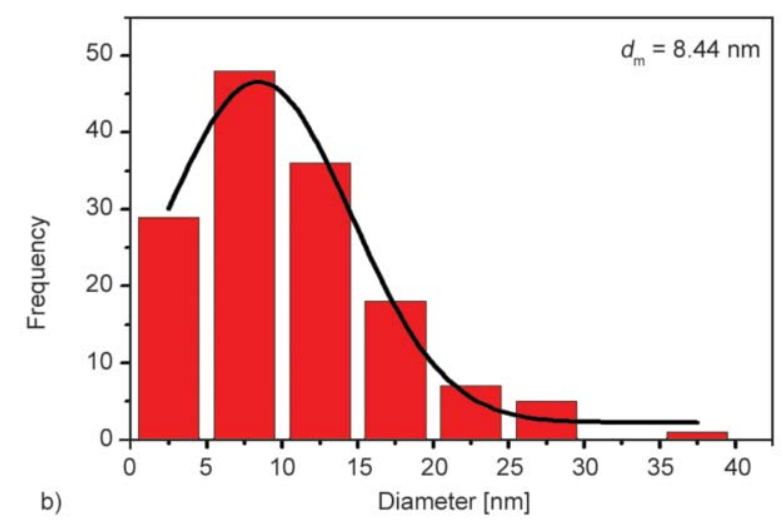

Figure 3. TEM high contrast image showing the presence of SiQD in polysilane (a); SiQDs size distribution histogram and fitted curve showing the average diameter $(b)$ 


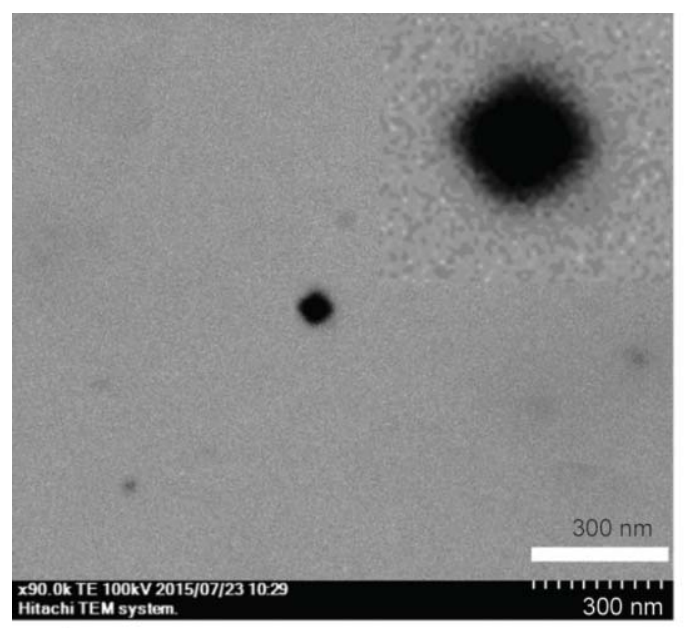

a)

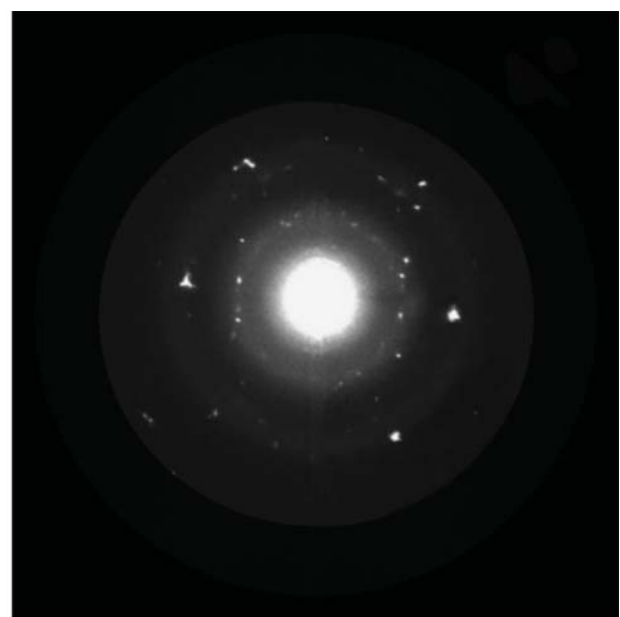

b)

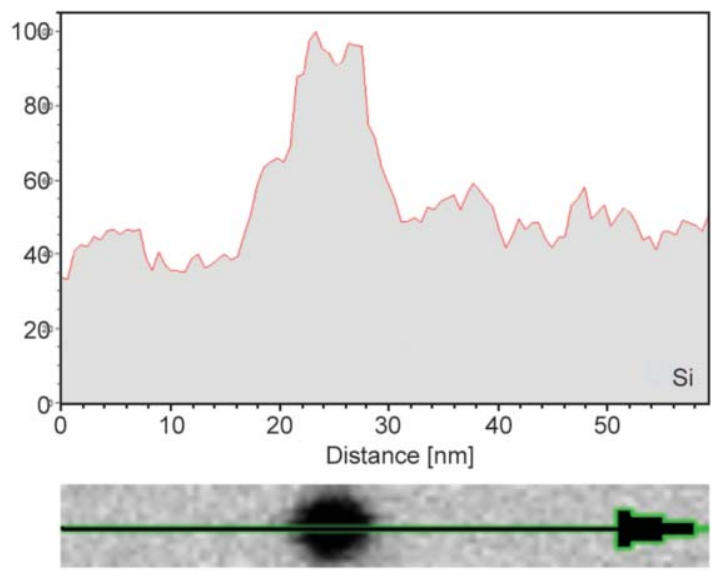

c)

Figure 4. STEM image in bright field detection showing the compact structure of SiQD (a); SAED pattern showing the characteristic three rings (b); EDX analysis, line profile comparison of the silicon content in SiQD versus background (c)

mentum transfer vector, $q$ [41]. In the region of small scattering angles, the form factor $P(q)$ can be approximated by a Gaussian curve. According to Guinier, this particular part of the curve is related to the overall size of the particles and can be used to calculate their radius of gyration $\left(R_{\mathrm{g}}\right)$ [42]. Thus, $R_{\mathrm{g}}$ is the slope determined by the strait-line fitting in the graphical representation of the $\ln (I)$ versus $q^{2}$ (Figure 6) according to Equation (1):

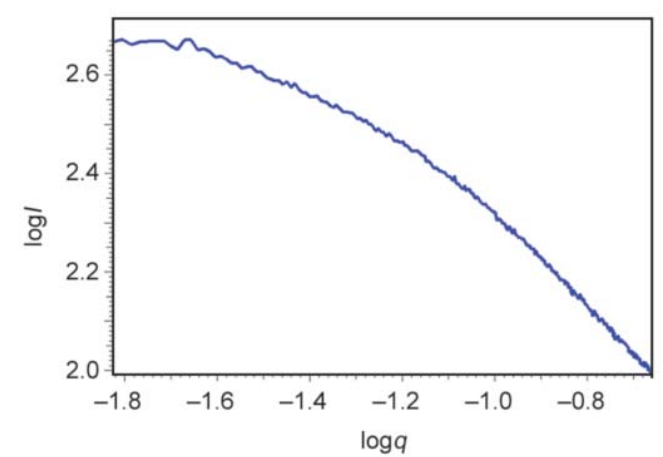

Figure 5. Scattering curve of NQD solution sample in logarithmic plot $\ln I(q)=\ln a_{0}-\frac{R_{\mathrm{g}}^{2} q^{2}}{3}$

$R_{\mathrm{g}}$ is the size parameter and it is model independent, and ao is the extrapolated zero angle intensity. The result obtained through this approach was $R_{\mathrm{g}}=$ $24.5 \AA$. Since the TEM-STEM analysis showed that the particles are quite homogeneous and of spherical shape, then the average diameter of the SiQDs in real

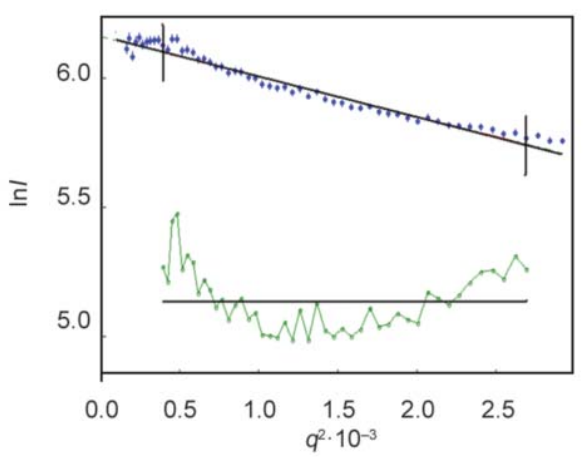

Figure 6. Guinier plot of the scattering intensity for NQD samples in solution 
space calculated using Equation (2) is $D_{\max }=$ $6.3 \mathrm{~nm}$.

$$
D_{\max }=2\left(\frac{5}{3}\right)^{1 / 2} R_{\mathrm{g}}
$$

Further interpretation of the SAXS results intended to tentatively reveal the origin of the high stability of the SiQDs colloidal solution. Such extremely stable colloidal dispersions of nanoparticles are normally obtained by using additional stabilizing agents. Because no stabilizing agents are necessary for the NQD dispersions, it was hypothesized that part of the polysilane chains are physically adsorbed onto the SiQDs surface, thus preventing their aggregation and separation.

In order to verify this hypothesis by SAXS, the model approach data analysis is usually done with the appropriate software. DIFFRAC ${ }^{\text {plus }}$ NanoFit is an interactive graphics-based, non-linear least-squares data analysis program for one-dimensional SAXS data. This data evaluation software attempts modelling of small-angle scattering data from systems showing short-range order only and isotropic scattering spectra, so that the scattering intensity is only a function of the modulus of the scattering vector. The 2D intensity distribution in reciprocal space is further processed in order to obtain the corresponding real-space structure. The model in which scatterers are spherical particles containing a dense core and an envelope of polymeric chains is a perfect fit for the aforementioned hypothesis. The mathematical expressions describing such a structure have been derived by Pedersen [43] see Equation (3):

$$
\begin{aligned}
& P(q)=\frac{1}{M^{2}}\left[\rho_{\mathrm{s}}^{2} F_{1}^{2}(q, R)+N_{\mathrm{c}} \rho_{\mathrm{c}}^{2} P_{\mathrm{GP}}^{2}(q, L)+\right. \\
& \left.+N_{\mathrm{c}}\left(N_{\mathrm{c}}-1\right) \rho_{\mathrm{c}}^{2} S_{\mathrm{cc}}(q)+2 N_{\mathrm{c}} \rho_{\mathrm{s}} \rho_{\mathrm{c}} S_{\mathrm{cc}}(q)\right]
\end{aligned}
$$

with $S_{\mathrm{cc}}(q)$ described by Equation (4):

$$
S_{\mathrm{cc}}(q)=F_{1}(q, R) \psi\left(q, R_{\mathrm{g}}\right)\left[\frac{\sin \left(q\left(R+d R_{\mathrm{g}}\right)\right)}{q\left(R+d R_{\mathrm{g}}\right)}\right]^{2}
$$

In this relation:

$M=\rho_{\mathrm{s}}+N_{\mathrm{c}} \rho_{\mathrm{c}}$ is the scattering mass, where $N_{\mathrm{c}}$ is the number of chains, $\rho_{\mathrm{s}}$ is the total excess scattering length of the core and $\rho_{\mathrm{c}}$ is the total excess scattering length of a corona chain. Non-penetration of the chains into the core region is mimicked by $d \approx 1$ for $R \gg R_{\mathrm{g}} ; R_{\mathrm{g}}$ is the root-mean-square radius of gyra- tion; $F_{1}(q, R)$ is the form factor amplitude of a homogeneous sphere; $P_{\mathrm{GP}}(q)$ is the form factor of a Gaussian chain; $\psi\left(q, R_{\mathrm{g}}\right)=[1-\exp (-u)] / u ; u=R_{\mathrm{g}}^{2} q^{2}$.

A fitting of the experimental scattering curve with the calculated plot (Figure 7) shows the perfect match with the proposed model. In addition, further insight on the internal structure of the scatterers has been obtained. Thus, the radius of the dense core of SiQDs was determined to be $R=2.8 \mathrm{~nm}$. Also, the radius of gyration corresponding to the polymeric chains adsorbed onto solid surface is $R_{\mathrm{c}}=1.6 \mathrm{~nm}$, which confirms the presence of polysilane onto the particle surface. Looking at the SiQDs diameter, as calculated by this model, one should observe that this value is quite similar to that obtained by the free model approach $\left[D_{\max (\text { model })}=2 \cdot 2.8=5.6 \mathrm{~nm}\right]$. The difference between the values obtained using each model comes from the polydispersity of the nanoparticles, which was not considered in the first method. In conclusion, SAXS results indicate that both the thin films and the solutions of NQD contain SiQDs as solid nanoparticles of spherical shape and sizes with an average maximum around $6 \mathrm{~nm}$. The SiQDs particles are covered by a thin layer of polymer of about $2 \mathrm{~nm}$ thick, which prevents SiQDs aggregation in solution and leads to highly stable colloidal dispersions.

Further experiments investigated the fluorescence of NQD in the presence of CTP.

Diaryl-substituted pyridine derivatives are bioisosteres of terpyridine that are known to exhibit a strong cytotoxicity against several human cancer cell lines through the inhibition of topoisomerase I [4448]. An analog of such diaryl-substituted pyridines (CTP) was synthesized using 3-dimethylamino-1(thiophen-2-yl)-1-propanone hydrochloride 1 (a ke-

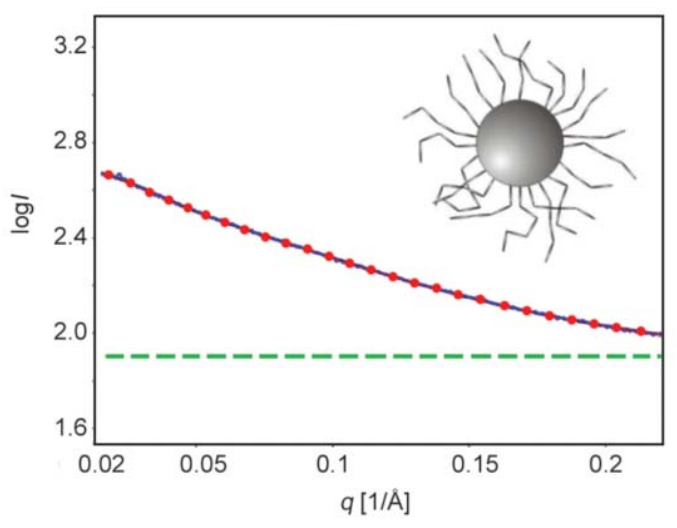

Figure 7. Correlation of the scattering curve between experimental and calculated (doted) showing the perfect mach with the assumed theoretical model 

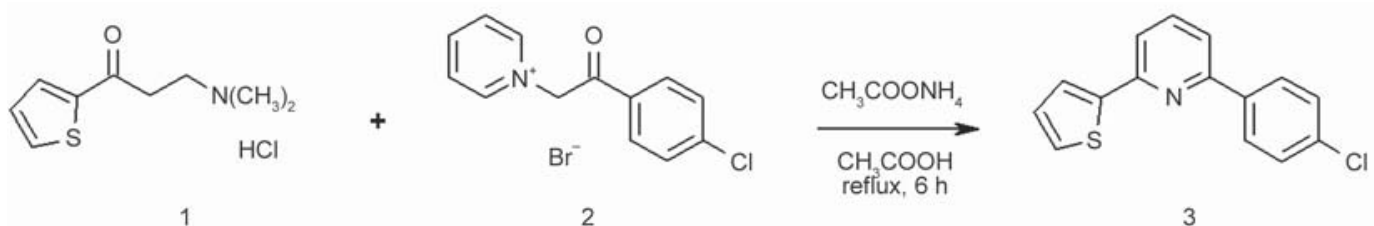

Figure 8. Synthesis of 2-(4-chlorophenyl)-6-(thiophen-2-yl)pyridine 3 (CTP)

tonic Mannich base), 4-chlorophenacylpyridinium bromide 2 and ammonium acetate as starting materials in an one-pot approach (Figure 8). The reaction sequence comprises the addition of the cycloimmonium salt to the $\alpha, \beta$-unsaturated ketone thermally generated in situ from the ketonic Mannich base to yield a 1,5-diketone, whose subsequent ring closure in the presence of ammonium acetate leads to the desired pyridine derivative [37]. Treatment of a chlocharcoal followed by recrystallization from ethanol chlorophenyl)-6-(thiophen-2-yl)pyridine 3 (CTP).

\subsection{Spectroscopy of CTP}

The CTP structure features an electron-acceptor moiety, namely the pyridine ring, linked to an electrondonor moiety, i.e. the thiophene ring. Starting from this prototype, similar molecules containing electron-donor and -acceptor units in their structure can be further tailored with a view to obtain lower bandgap, broader absorption, and higher mobility of charges. As the number of reports dealing with the florescence of CTP analogs is scarce, we first examined the photophysical properties of this compound. The study was also required in order to understand the origin of the electronic transitions involved in the emission process.

The UV-vis absorption spectrum of CTP as $0.01 \%$ solution in xylenes is shown in Figure 9. The compound has a broad and intense absorption in the range of 290 to $350 \mathrm{~nm}$. The profile of the absorption pres-

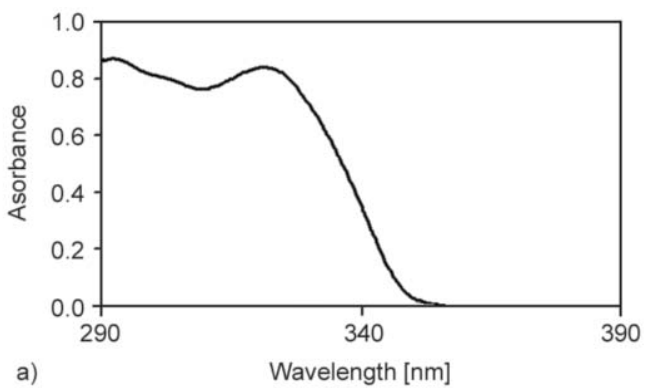
roform solution of the crude reaction product with of the recovered solid afforded colourless 2-(4-

ents a well-defined maximum at $321 \mathrm{~nm}$, a barely visible shoulder at $302 \mathrm{~nm}$ and finally a large wide shoulder centered at $292 \mathrm{~nm}$. The absorption maximum located at $302 \mathrm{~nm}$ could be assigned to the $\pi-\pi^{*}$ electron transitions within the conjugated thiophenepyridine system. On the other hand, the $321 \mathrm{~nm}$ absorption band results from an intramolecular charge transfer which is specific for fluorescent compounds with electron-donor and -acceptor units in their structure.

The emission spectrum of CTP was measured by excitation around the determined absorption maxima. Irrespective of the excitation wavelength, the corresponding fluorescence (FL) spectrum showed only a single emission band centered at $368 \mathrm{~nm}$. The highest intensity was obtained for an excitation wavelength of $321 \mathrm{~nm}$, and this band was assigned to the emission of the first singlet excited state, S1 (fluorescence).

\subsection{Spectroscopy of NQD}

Polyhydrosilane presents in its structure numerous phenyl rings that are linked directly to the silicon backbone of the copolymer. The strong effects due to interactions at the level of the excited states between these aromatic rings and the silicon-based chain of the copolymer will determine the material's photophysical properties, which could be further exploited at molecular level. The UV-vis absorption spectrum of NQD is presented in Figure 10. As expected, a large shoulder is noticeable at approximately $310 \mathrm{~nm}$, and its presence in the spectrum has been

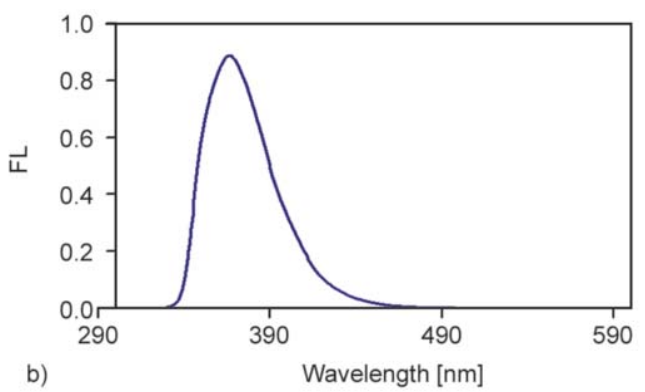

b)

Figure 9. UV-vis absorption spectrum of CTP (a); FL spectrum of CTP. Excitation was done at $321 \mathrm{~nm}$, (b) 

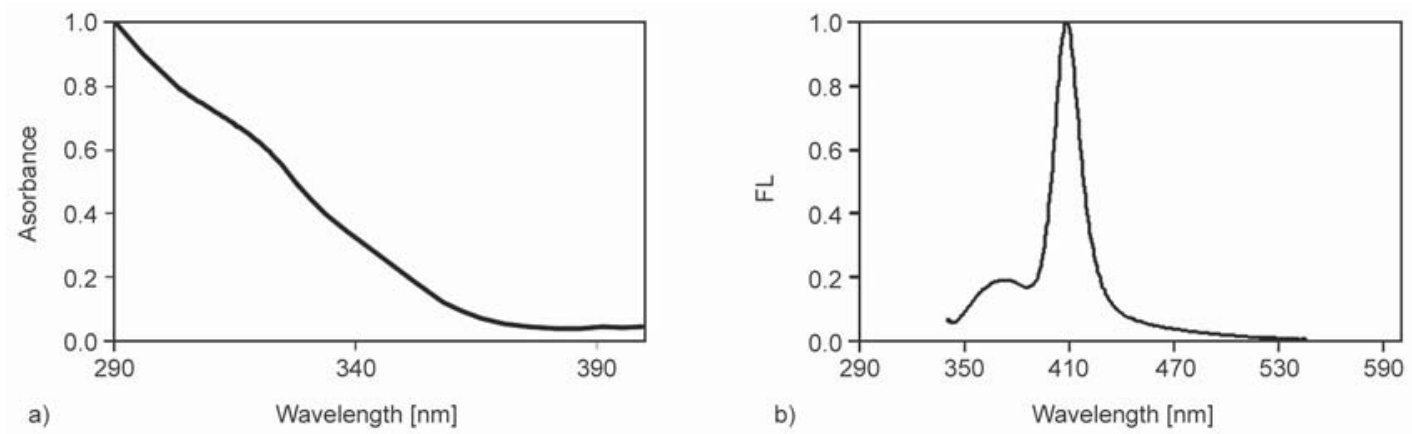

Figure 10. UV-vis spectrum of NQD (a); FL spectra of NQD. Excitation at $330 \mathrm{~nm}$, (b)

associated with the transitions of the $\sigma$ electrons in the polyhydrosilane matrix. A characteristic feature of polyhydrosilanes is the low resolution of the UV absorption maximum compared with other polysilanes structures. This specific profile is a consequence of the additional segmentation of the linear conjugated chain induced by the more flexible methylhydrosilyl moieties [36, 40, 49].

Excitation around the absorption maximum wavelength leads to the emission profile displayed in Figure 10b. The FL spectrum presented two emission bands. First, there is an expected broad emission band at $370 \mathrm{~nm}$ that was assigned to the $\sigma-\sigma^{*}$ electrons relaxation specific for the $\sigma$ conjugated polysilanes. Second, another narrow emission peak with a much higher intensity is present at $410 \mathrm{~nm}$. According to previous reports, this emission is characteristic for the SiQDs with dimensions below $10 \mathrm{~nm}$ $[50,51]$. It is important to note that the highest intensity of the SiQDs emission was obtained by excitation at $330 \mathrm{~nm}$, the same wavelength as for the polysilane structure.

\subsection{Effect of CTP on NQD}

UV-vis analysis of colloidal NQD samples having concentrations of CTP within $0.1-1 \%$ range has been performed with a view to establish the working limit.

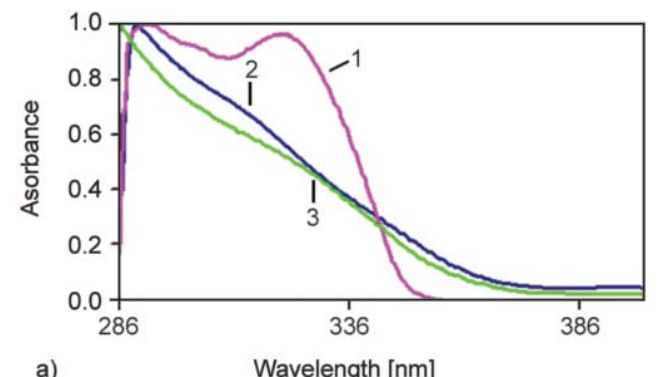

Thus, it was noted that for concentrations higher than $0.2 \%$, the stronger absorption of CTP totally conceals the absorption of NQD. Below this value, the UV absorption spectrum shows elements common to both NQD and CTP UV spectra (Figure 11). On one hand, the overall aspect of the UV spectrum of stained NQD is similar to that of the pristine NQD, having just one large, barely noticeable shoulder. On the other hand, the absorption maximum recorded for stained NQD has shifted from $310 \mathrm{~nm}$ (as it was in the case of NQD) to $321 \mathrm{~nm}$ (as it was recorded for CTP). The probable cause of these modifications is the overlapping of electron transitions having similar energy gap for both NQD and CTP, which in turn affects the corresponding excited states. Interaction of polyhydrosilanes with the neighbouring electroactive species is a consequence of the intramolecular weak charge-transfer structures along the unshielded methyhydrosilyl segments [52]. The presence of CTP molecules in the immediate vicinity of polyhydrosilane leads to an extension of the $\sigma$ electrons conjugated system through coupling with the dual $\pi$ conjugated system of CTP. In addition, the presence of phenyl groups attached to the polysilane backbone contributes to a lowering of the band gap energy through $\sigma-\pi$ conjugation. This also generates particular excited states, which are involved in electron

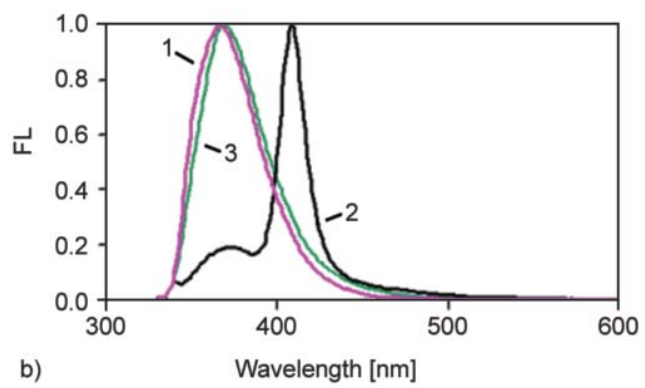

Figure 11. (a) Comparison between the UV absorption spectra of CTP (1), NQD (2) and stained NQD (3); (b) Comparison between the FL emission spectra of CTP (1), NQD (2) and stained NQD (3) 
transition processes at both intra- and intermolecular levels [21].

The FL spectrum of stained NQD shows a single emission band at $370 \mathrm{~nm}$ for excitation wavelengths within the 310-330 $\mathrm{nm}$ range (Figure 11b). Interestingly, the emission profile of stained NQD is similar to that of pristine CTP, and not to the emission profile of NQD.

Another important observation is that a very small concentration of TCP $\left(0.33 \cdot 10^{-7} \mathrm{M}\right)$ induced the suppression of SiQDs fluorescence emission up to $29.7 \%$, meanwhile upon addition of $2.33 \cdot 10^{-7} \mathrm{M}$ the fluorescence is quenched with $76.4 \%$ (Figure 12a).

Generally, the fluorescence quenching represents the decrease of the quantum yield of fluorescence from the fluorophore induced by different molecular interactions with the quencher molecule (energy transfer, excited-state reaction, molecules rearrangement, ground state complex formation, collision quenching) and also the quenching mechanism can be static or dynamic. Here, in order to elucidate the mechanism of quenching, we employed the well-known SternVolmer Equation (5) and modified Stern-Volmer Equation (6) [53]:

$\frac{I_{0}}{I}=1+K_{\mathrm{SV}}[Q]$
$\frac{I_{0}}{\left(I_{0}-I\right)}=\frac{1}{f_{\mathrm{a}}}+\frac{1}{f_{\mathrm{a}} k_{\mathrm{a}}[Q]}$

where $I_{0}$ and $I$ are the values of initial fluorescence intensities and in the presence of a quencher, respectively, $[Q]$ is the quencher concentration, $K_{\mathrm{SV}}$ is the Stern-Volmer dynamic quenching constant, ka is the fraction of accessible fluorescence and $f_{\mathrm{a}}$ is the effected quenching constant for the accessible fluorophores.

The Stern-Volmer plot of NQD in presence of TCP is shown in Figure 12b. It could be noted that the plot is linear, indicating that the quenching mechanism is probably single quenching $[53,54]$. Moreover, the curve of $I_{0} /\left(I_{0}-I\right)$ versus $1 /[Q]$ was also linear for the TCP concentrations used in the study (Figure 12c). Both these obviously represent characteristics of a static quenching mechanism $[53,55]$.

A system exhibiting this behavior could be used to build multi-channel sensing devices. Thus, the NQDbased sensor will emit an intense signal at $370 \mathrm{~nm}$ when in contact with trace amounts of CTP. In the absence of CTP, a different signal will be emitted at $410 \mathrm{~nm}$. Both signals can be generated using the same excitation wavelength, namely $330 \mathrm{~nm}$. In any of these cases, the emission could be collected and used to trigger a specific response. This approach
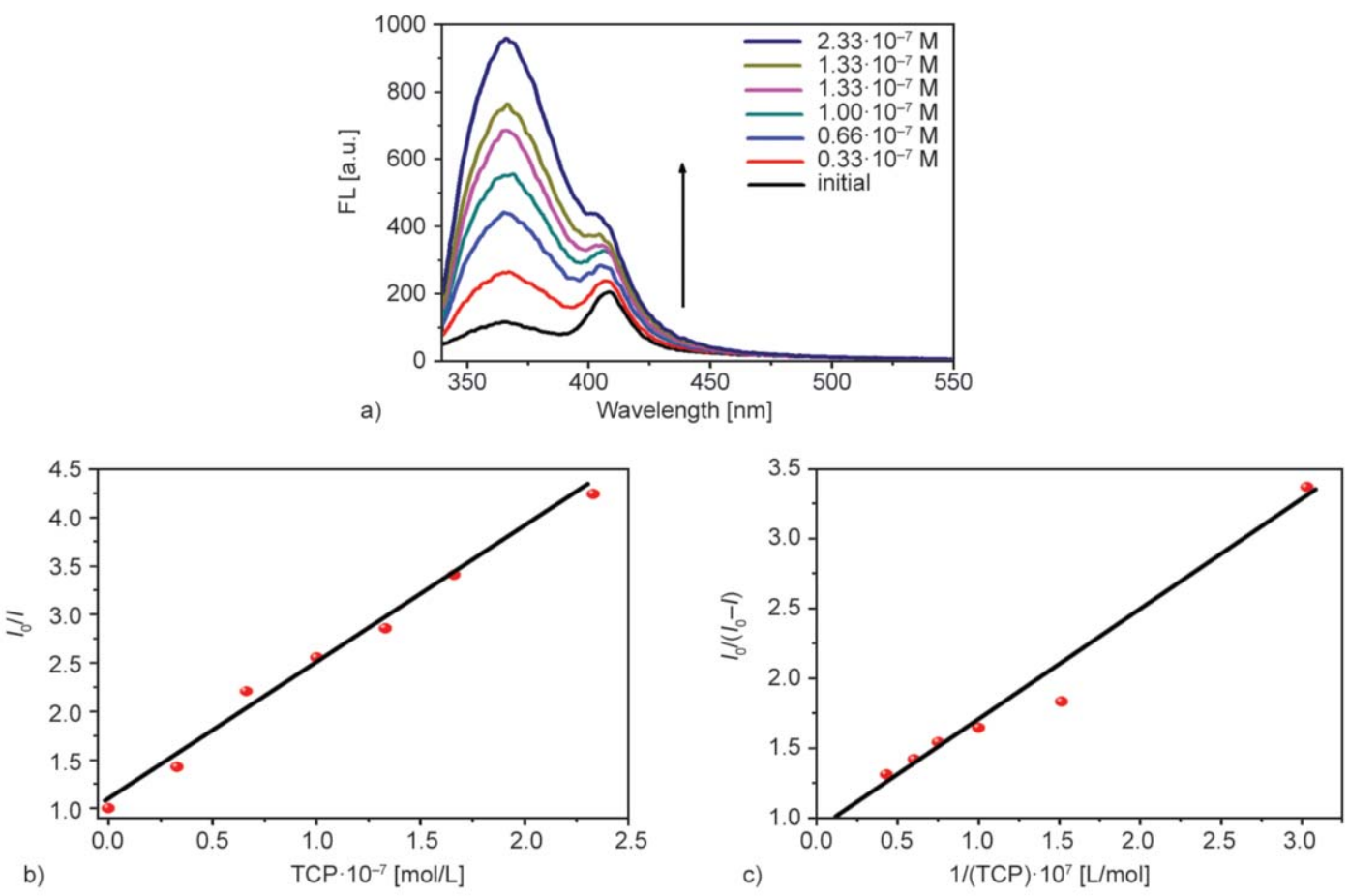

Figure 12. Influence of TCP on the fluorescence intensity of SiQDs (a); Stern-Volmer plot (b) and modified Stern-Volmer plot (c) showing the concentration effect of TCP 
opens new opportunities to develop complex, high resolution imaging/sensing systems designed to increase anticancer therapy efficiency.

\section{Conclusions}

This work describes the sensing properties of a nanocomposite obtained by microwaves-assisted Wurtz coupling of methyldichlorosilane with diphenyldichlorosilane. The material is a solid powder containing SiQDs dispersed in a polysilane matrix soluble in usual organic solvents. Upon dissolution, indefinitely stable colloidal solutions of SiQDs are obtained. Thin films cast from such solutions were analyzed by TEM, and showed the presence of SiQDs dispersed within the polysilane thin film. The remarkable stability of the NQDs colloidal dispersion has been explained using SAXS. Thus, it was found that the spherical silicon nanoparticles are covered with a thin layer of polymer that prevents their aggregation and sedimentation. Further experiments explored the changes induced to the fluorescence of colloidal SiQDs by the presence of CTP, a potential cytotoxic agent. It was found that the SiQDs strong emission is totally quenched if low amounts of CTP are present. Since CTP has his own fluorescence, its combination with colloidal SiQDs results in a dual channel sensing system activated by a single excitation wavelength. The system could find application in tumour imaging and therapy.

\section{References}

[1] Berger C., Song Z., Li T., Li X., Ogbazghi A. Y., Feng R., Dai Z., Marchenkov A. N., Conrad E. H., First P. N., de Heer W. A.: Ultrathin epitaxial graphite: 2D electron gas properties and a route toward graphene-based nanoelectronics. Journal of Physical Chemistry B, 108, 19912-19916 (2004). DOI: $10.1021 / j p 040650 f$

[2] Banerjee S. K., Register L. F., Tutuc E., Reddy D., MacDonald A. H.: Bilayer pseudospin field-effect transistor (BiSFET): A proposed new logic device. IEEE Electron Device Letters, 30, 158-160 (2009). DOI: 10.1109/LED.2008.2009362

[3] Cavin R. K., Zhirnov V. V., Herr D. J. C., Avila A., Hutchby J.: Research directions and challenges in nanoelectronics. Journal of Nanoparticle Research, 8, 841-858 (2006). DOI: $10.1007 / \mathrm{s} 11051-006-9123-4$

[4] Li L., Deng L.: Random lasers in dye-doped polymerdispersed liquid crystals containing silver nanoparticles. Physica B: Condensed Matter, 407, 4826-4830 (2012). DOI: $10.1016 /$ j.physb.2012.09.023
[5] Skirtach A. G., Dejugnat C., Braun D., Susha A. S., Rogach A. L., Parak W. J., Möhwald H., Sukhorukov G. B.: The role of metal nanoparticles in remote release of encapsulated materials. Nano Letters, 5, 1371-1377 (2005).

DOI: $10.1021 / \mathrm{n} 1050693 \mathrm{n}$

[6] Li S., Lin M. M., Toprak M. S., Kim D. K., Muhammed M.: Nanocomposites of polymer and inorganic nanoparticles for optical and magnetic applications. Nano Reviews, 1, 5214/1-5214/19 (2010).

DOI: $10.3402 /$ nano.v1i0.5214

[7] Ahirwal G. K., Mitra C. K.: Direct electrochemistry of horseradish peroxidase-gold nanoparticles conjugate. Sensors, 9, 881-894 (2009).

DOI: $10.3390 / \mathrm{s} 90200881$

[8] Murray R. W.: Nanoelectrochemistry: Metal nanoparticles, nanoelectrodes, and nanopores. Chemical Reviews, 108, 2688-2720 (2008).

DOI: $10.1021 / \mathrm{cr} 068077 \mathrm{e}$

[9] Gómez-Herrero J., Zamora F.: Coordination polymers for nanoelectronics. Advanced Materials, 23, 53115317 (2011).

DOI: 10.1002/adma.201101952

[10] Han J., Ji Q., Qiu S., Li H., Zhang S., Jin H., Li Q.: A versatile approach to obtain a high-purity semiconducting single-walled carbon nanotube dispersion with conjugated polymers. Chemical Communications, 51, 4712-4714 (2015).

DOI: $10.1039 / \mathrm{C} 5 \mathrm{CC} 00167 \mathrm{~F}$

[11] Gui R., Jin H., Wang Z., Tan L.: Recent advances in synthetic methods and applications of colloidal silver chalcogenide quantum dots. Coordination Chemistry Reviews, 296, 91-124 (2015). DOI: $10.1016 /$ j.ccr.2015.03.023

[12] Knoblauch C., Griep M., Friedrich C.: Recent advances in the field of bionanotechnology: An insight into optoelectric bacteriorhodopsin, quantum dots, and noble metal nanoclusters. Sensors, 14, 19731-19766 (2014). DOI: $10.3390 / \mathrm{s} 141019731$

[13] Kodriano Y., Schmidgall E. R., Benny Y., Gershoni D.: Optical control of single excitons in semiconductor quantum dots. Semiconductor Science and Technology, 29, 053001/1-053001/24 (2014). DOI: $10.1088 / 0268-1242 / 29 / 5 / 053001$

[14] Holmström P., Thylén L.: Electro-optic switch based on near-field-coupled quantum dots. Applied Physics A, 115, 1093-1101 (2014). DOI: $10.1007 / \mathrm{s} 00339-014-8420-5$

[15] Cheng X., Hinde E., Owen D. M., Lowe S. B., Reece P. J., Gaus K., Gooding J. J.: Enhancing quantum dots for bioimaging using advanced surface chemistry and advanced optical microscopy: Application to silicon quantum dots (SiQDs). Advanced Materials, 27, 61446150 (2015). DOI: $10.1002 /$ adma.201503223 
[16] Montalti M., Cantelli A., Battistelli G.: Nanodiamonds and silicon quantum dots: Ultrastable and biocompatible luminescent nanoprobes for long-term bioimaging. Chemical Society Reviews, 44, 4853-4921 (2015). DOI: $10.1039 / \mathrm{C} 4 \mathrm{CS} 00486 \mathrm{H}$

[17] Campos B. B., Algarra M., Alonso B., Casado C. M., Jiménez-Jiménez J., Rodríguez-Castellón E., Esteves da Silva J. C. G.: Fluorescent sensor for Cr(VI) based in functionalized silicon quantum dots with dendrimers. Talanta, 144, 862-867 (2015).

DOI: $10.1016 /$ j.talanta.2015.07.038

[18] Peng X., Long Q., Li H., Zhang Y., Yao S.: 'Turn on-off' fluorescent sensor for protamine and heparin based on label-free silicon quantum dots coupled with gold nanoparticles. Sensors and Actuators B: Chemical, 213, 131-138 (2015)

DOI: $10.1016 /$ j.snb.2015.02.070

[19] Brus L.: Luminescence of silicon materials: Chains, sheets, nanocrystals, nanowires, microcrystals, and porous silicon. Journal of Physical Chemistry, 98, 3575 3581 (1994).

DOI: $10.1021 / \mathrm{j} 100065 \mathrm{a} 007$

[20] Kitao T., Bracco S., Comotti A., Sozzani P., Naito M., Seki S., Uemura T., Kitagawa S.: Confinement of single polysilane chains in coordination nanospaces. Journal of the American Chemical Society, 137, 5231-5238 (2015). DOI: $10.1021 /$ jacs.5b02215

[21] Sacarescu L., Kostromin S., Bronnikov S.: Synthesis and properties of polydiphenylsilane/fullerene $\mathrm{C}_{60}$ nanocomposites. Materials Chemistry and Physics, 149150, 430-436 (2015).

DOI: 10.1016/j.matchemphys.2014.10.041

[22] Okazaki A., Akita S., Nakayama Y.: Nanolithography of organic polysilane films using carbon nanotube tips - Application to the etching process. Physica B: Condensed Matter, 323, 151-152 (2002).

DOI: $10.1016 / \mathrm{S} 0921-4526(02) 00884-0$

[23] Srinivasan R.: Ablation of polymers and biological tissue by ultraviolet lasers. Science, 234, 559-565 (1986). DOI: $10.1126 /$ science. 3764428

[24] Miller R. D., Wallraff G. M., Clecak N., Sooriyakumaran R., Michl J., Karatsu T., McKinley A. J., Klingensmith K. A., Downing J.: Polysilanes: Photochemistry and deep UV lithography. Polymer Engineering and Science, 29, 882-886 (1989).

DOI: $10.1002 /$ pen.760291311

[25] Hasegawa T., Iwasa Y., Sunamura H., Koda T., Tokura Y., Tachibana H., Matsumoto M., Abe S.: Nonlinear optical spectroscopy on one-dimensional excitons in silicon polymer, polysilane. Physical Review Letters, 69, 668-671 (1992).

DOI: 10.1103/PhysRevLett.69.668

[26] Tang H., Liu Y., Huang B., Qin J., Fuentes-Hernandez C., Kippelen B., Li S., Ye C.: Synthesis and optical properties of a series of chromophore functionalized polysilanes. Journal of Materials Chemistry, 15, 778-784 (2005).

DOI: $\underline{10.1039 / B 413016 B}$
[27] Tsukuda S., Seki S., Saeki A., Kozawa T., Tagawa S., Sugimoto M., Idesaki A., Tanaka S.: Precise control of nanowire formation based on polysilane for photoelectronic device application. Japanese Journal of Applied Physics, 43, 3810-3814 (2004).

DOI: $10.1143 / \mathrm{JJAP} .43 .3810$

[28] Ardeleanu R., Mangalagiu I., Sacarescu G., Simionescu M., Sacarescu L.: Polysilane-cycloimmonium salts: New materials for organic semiconductors. Macromolecular Rapid Communications, 25, 1231-1235 (2004). DOI: $10.1002 /$ marc.200400115

[29] Masuda T., Matsuki Y., Shimoda T.: Pyrolytic transformation from polydihydrosilane to hydrogenated amorphous silicon film. Thin Solid Films, 520, 6603-6607 (2012). DOI: $10.1016 /$ j.tsf.2012.07.028

[30] Iyer G. R. S., Hobbie E. K., Guruvenket S., Hoey J. M., Anderson K. J., Lovaasen J., Gette C., Schulz D. L., Swenson O. F., Elangovan A., Boudjouk P.: Solutionbased synthesis of crystalline silicon from liquid silane through laser and chemical annealing. ACS Applied Materials and Interfaces, 4, 2680-2685 (2012).

DOI: $10.1021 / \mathrm{am} 300334 \mathrm{p}$

[31] Shimoda T., Matsuki Y., Furusawa M., Aoki T., Yudasaka I., Tanaka H., Iwasaki H., Wang D., Miyasaka M., Takeuchi Y.: Solution-processed silicon films and transistors. Nature, 440, 783-786 (2006). DOI: 10.1038 /nature04613

[32] Sacarescu G., Sacarescu L., Ardeleanu R., Kurcok P., Jedliński Z.: Si-H functional polysilanes via a homogeneous reductive coupling reaction. Macromolecular Rapid Communications, 22, 405-408 (2001). DOI: 10.1002/1521-3927(20010301)22:6<405::AIDMARC405>3.0.CO;2-N

[33] Sacarescu L., Ardeleanu R., Sacarescu G., Simionescu M., Hurduc N.: Polydiphenylsilanes bearing photosensitive azocrown groups. Chemical Communications, 2006, 788-789 (2006). DOI: $10.1039 / \mathrm{B} 514161 \mathrm{C}$

[34] Sacarescu L., Ardeleanu R., Sacarescu G., Simionescu M., Mangalagiu I.: Polysilane-metal complexes for organic semiconductors. High Performance Polymers, 19, 501-509 (2007).

DOI: $10.1177 / 0954008306081193$

[35] Sacarescu L., Siokou A., Ardeleanu R., Sacarescu G., Simionescu M.: Low-temperature restructuring processes in polyhydrosilanes. Macromolecular Rapid Communications, 27, 1054-1059 (2006).

DOI: $10.1002 /$ marc. 200600192

[36] Sacarescu L., Bockholt A., Siokou A., Simionescu M., Sacarescu G.: Structural and optical properties of polyhydrosilanes. Macromolecular Chemistry and Physics, 210, 2015-2021 (2009).

DOI: $10.1002 / \mathrm{macp} .200900384$

[37] Roman G.: Generation of a structurally diverse library through alkylation and ring closure reactions using 3dimethylamino-1-(thiophen-2-yl)propan-1-one hydrochloride. Acta Chimica Slovenica, 60, 70-80 (2013). 
[38] Sacarescu G., Simionescu M., Harabagiu V., Sacarescu L.: Microwave-assisted Wurtz coupling of methylphenyldichlorosilane in solvent-free conditions. e-Polymers 12, 145-150 (2012).

DOI: $10.1515 /$ epoly.2012.12.1.145

[39] Sacarescu L., Siokou A., Sacarescu G., Simionescu M., Mangalagiu I.: Methylhydrosilyl chemostructural effects in polyhydrosilanes. Macromolecules, 41, 1019-1024 (2008). DOI: $10.1021 / \mathrm{ma} 071853 \mathrm{f}$

[40] Konarev P. V., Volkov V. V., Sokolova A. V., Koch M. H. J., Svergun D. I.: PRIMUS: a Windows PC-based system for small-angle scattering data analysis. Journal of Applied Crystallography, 36, 1277-1282 (2003). DOI: $10.1107 /$ S0021889803012779

[41] Glatter O., Kratky O.: Small angle X-ray scattering. Academic Press, London (1982).

[42] Guinier A., Fournet G.: Small-angle scattering of Xrays. Wiley, New York (1955).

[43] Lindner P., Zemb T.: Neutrons, X-rays and light. Scattering methods applied to soft condensed matter. Elsevier, Amsterdam (2002).

[44] Kim D. S. H. L., Ashendel C. L., Zhou Q., Chang C-T., Lee E-S., Chang C-J.: Novel protein kinase $\mathrm{C}$ inhibitors: $\alpha$-terthiophene derivatives. Bioorganic and Medicinal Chemistry Letters, 8, 2695-2698 (1988). DOI: $10.1016 / \mathrm{S} 0960-894 \mathrm{X}(98) 00468-5$

[45] Zhao L-X., Kim T. S., Ahn S-H., Kim T-H., Kim E-K., Cho W-J., Choi H., Lee C-S., Kim J-A., Jeong T. C., Chang C-J., Lee E-S.: Synthesis, topoisomerase I inhibition and antitumor cytotoxicity of $2,2^{\prime}: 6^{\prime}, 2^{\prime \prime}-, 2,2^{\prime}: 6^{\prime}, 3^{\prime \prime}-$ and 2,2':6',4'"-terpyridine derivatives. Bioorganic and Medicinal Chemistry Letters, 11, 2659-2662 (2001). DOI: 10.1016/S0960-894X(01)00531-5

[46] Zhao L-X., Moon Y-S., Basnet A., Kim E-K., Jahng Y., Park J. G., Jeong T. C., Cho W-J., Choi S-U., Lee C. O., Lee S-Y., Lee C-S., Lee E-S.: Synthesis, topoisomerase I inhibition and structure-activity relationship study of 2,4,6-trisubstituted pyridine derivatives. Bioorganic and Medicinal Chemistry Letters, 14, 1333-1337 (2004).

DOI: $10.1016 / \mathrm{j} . \mathrm{bmcl} .2003 .11 .084$
[47] Zhao L-X., Sherchan J., Park J. K., Jahng Y., Jeong BS., Jeong T. C., Lee C-S., Lee E-S.: Synthesis, cytotoxicity and structure-activity relationship study of terpyridines. Archives of Pharmacal Research, 29, 1091-1095 (2006).

DOI: $10.1007 / \mathrm{BF} 02969297$

[48] Basnet A., Thapa P., Karki R., Na Y., Jahng Y., Jeong BS., Jeong T. C., Lee C-S., Lee E-S.: 2,4,6-Trisubstituted pyridines: Synthesis, topoisomerase I and II inhibitory activity, cytotoxicity, and structure-activity relationship. Bioorganic and Medicinal Chemistry, 15, 43514359 (2007).

DOI: 10.1016/j.bmc.2007.04.047

[49] Tarabukina E., Krasova A., Filippov A., Sacarescu L., Simionescu M., Sacarescu G., Soroceanu M., Harabagiu V.: Hydrodynamic and molecular characteristics of organosilane copolymers of low molecular weight. High Performance Polymers, 25, 79-86 (2013).

DOI: $10.1177 / 0954008312456890$

[50] Baldwin R. K., Pettigrew K. A., Ratai E., Augustine M. P., Kauzlarich S. M.: Solution reduction synthesis of surface stabilized silicon nanoparticles. Chemical Communications, 2002, 1822-1823 (2002).

DOI: $10.1039 / \mathrm{B} 205301 \mathrm{~B}$

[51] Kim T-Y., Park N-M., Kim K-H., Sung G. Y., Ok Y-W., Seong T-Y., Choi C-J.: Quantum confinement effect of silicon nanocrystals in situ grown in silicon nitride films. Applied Physics Letters, 85, 5355-5357 (2004). DOI: $10.1063 / 1.1814429$

[52] Sacarescu L., Simionescu M., Sacarescu G., Hitruc E. G.: Photocatalytic synthesis of silver nanoparticles using polysilane initiator. Journal of Nanoparticle Research, 13, 997-1005 (2010). DOI: $10.1007 / \mathrm{s} 11051-010-0120-2$

[53] Lakowicz J. R.: Principles of fluorescence spectroscopy. Springer, New York (2006).

[54] Zhang H., Wang Y., Fei Z., Wu L., Zhou Q.: Characterization of the interaction between Fe(III)-2,9,16,23tetracarboxyphthalocyanine and blood proteins. Dyes and Pigments, 78, 239-247 (2008). DOI: 10.1016/j.dyepig.2007.12.011

[55] Ware W. R.: Oxygen quenching of fluorescence in solution: An experimental study of the diffusion process. Journal of Physical Chemistry B, 66, 445-458 (1962). DOI: $10.1021 / \mathrm{j} 100809 \mathrm{a} 020$ 